\title{
The path switching surge protection system for telecommunications equipment
}

\author{
Wansung Kwon ${ }^{\mathrm{a}}$, Yongsu Kim ${ }^{\mathrm{b}}$, Dongmin Kim ${ }^{\mathrm{b}}$, Jongyeon Kim ${ }^{\mathrm{b}}$, Dongjin Kim ${ }^{\mathrm{c}}$ \\ ${ }^{a}$ Sunkwang LTI. INC., 15, Yangnyeong-ro, Gwanak-gu, Seoul and 08752, Republic of Korea. \\ ${ }^{b}$ Sunkwang LTI. INC., 30, Geumgyedong-gil, Janggye-ri, Hwaseong-myeon, Cheongyang-gun, Chungcheongnam-do and 33311, \\ Republic of Korea. \\ ${ }^{c}$ Sunkwang LTI. INC., 231, Dogok-ro, Gangnam-gu, Seoul and 06230, Republic of Korea.
}

\begin{abstract}
Existing surge protective device for communication loses its intrinsic characteristics by the system generated from inside/outside for its major elements in MOV (Metal Oxide Varistor), GDT (Gas Discharge Tube), TVS (Transient Voltage Suppressor) and resistance for passive elements. The surge protective device of the communication system is inserted directly to the middle of the line in the structure that, if it loses the function, normal protection on the protected equipment would be impossible and it generates direct damage to the protective equipment by the excessive voltage/current. Therefore, this paper describes on surge protective system for stable protection and easy maintenance of the communication system by periodically confirming the line condition and improvement of structure in double for the single protection type in series as the most vulnerable part.
\end{abstract}

Keywords: Surge; protection; SPD; telecommunications; parallel-type

\section{Introduction}

With the recent advancement of semiconductor technology for building intelligence, expansion of various control system and so forth with broad use in electric and electronic products, the damages from the surge are rapidly increasing. As the integration of the semiconductor enlarged, the circuit width inside the semiconductor narrowed. And outstanding conduction materials are used to operate in low voltage that the highest figure of the short-circuit is lowered that the system with many semiconductors weakens from the internal pressure and it is more vulnerable to the surge in proportion [1], [2].

The data/communication equipment is extremely vulnerable to the surge. As components get smaller, the operating voltage is lowered with the insulation voltage is significantly lowered and the TTL driver inside the RS-232 circuit may be broken down in the 40[V] surge and the CMOS chip has the breaking down in $17[\mathrm{~V}]$.

The surge is flowing in through the communication lines of telecommunication line, exclusively leased cable, axial cable, RS-232, RS-422, RS-485 and etc. There are many causes for surge but most surges appearing in communication line are induced through the communication line near to the lightning area. In addition, the surge is generated to the communication lines by the power line generated from the industrial facilities, such as, operating/suspension and etc. of the motor.

The surge protective device is installed to prevent the malfunction from lightning and damage on voltage, but it is not completely protected. Existing protective device is difficult to confirm in real time even if there is any damage or breakdown after the surge penetration and inspection and maintenance are not easily available [3].

\footnotetext{
* Manuscript received November 1, 2016; revised April 10, 2017.

Corresponding author. Tel.: 82-2-872-1500; E-mail address: wskwon@i-sk.com.

doi: $10.12720 /$ sgce.6.2.84-89
} 
For the case of the communication system, the protective device has the characteristics to connect in series that it has the risk of having the surge directly flowing into the protected equipment if the protective capability is lost by the damage to the protective device, and it may have the secondary damage for suspending the operation of the communication system with the personal accident or short-circuit in tracks.

In order to supplement problems in the existing surge protective device, this paper describes the surge protective system that applied the transfer method of parallel type to operate another protective device with the transfer system when the protective device is damaged by malfunctioning of surge, voltage or the like as one surge protective system operating at normal times.

\section{Structure and Operation of the Surge Protection System}

\subsection{Structure of system}

In general, in the event that the surge protective system is installed to the signal and communication system, it protects the equipment by connecting in series to the communication system which is different from the power connected in parallel that the breakdown of the protective system may directly inflict damages to the equipment.

In order to supplement the problem of such series structure, the parallel structure is facilitated to maintain the continuity of the protective capability. The transfer switch is applied to have the current flowing to the basic line, and if the basic line is broken down, the current is made to flow into another line to protect the protected equipment.

The proposed protective system is the combination of GDT, TVS, and resistance to be the basic protective circuit and two protective circuits are applied in parallel as shown in Fig. 1. The MCU for confirming the status of protective circuit and the structure for protective circuit transfer are additionally used. A photograph of the proposed protection system is shown in Fig. 2.

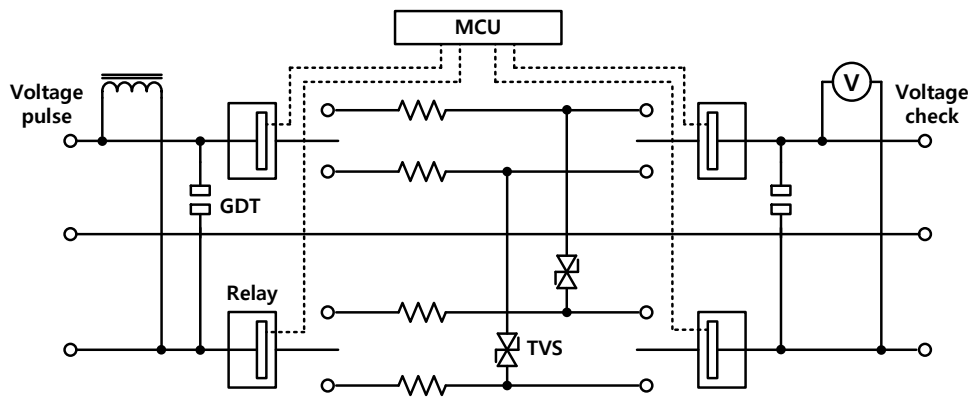

Fig. 1. Circuit diagram of the system.

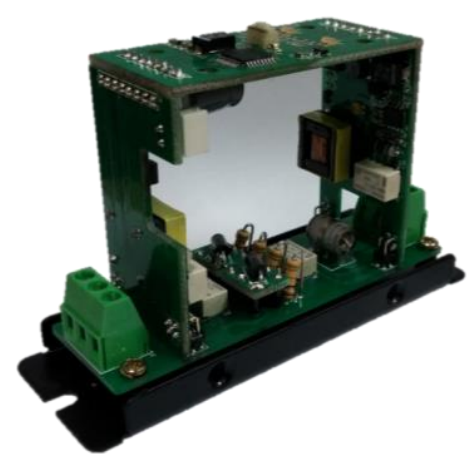

Fig. 2. Photo of the system. 


\subsection{Characteristics of operation}

It is not just applying two protective circuits in parallel, but to apply the functions for transfer depending on the condition of the protective circuit. For this purpose, the malfunctioning current like the surge is induced with the normal protective circuit with the feedback of the protective circuit condition not to have direct influence on the protected equipment.

The condition of protective circuit is classified in 4 modes and the information for each condition is defined as shown in Table 1.

- Mode 0: Confirm the voltage(Vin) status

- Mode 1: Confirm the protective circuit A operation

- Mode 2: Confirm the protective circuit B operation

- Mode 3: Confirm the loss of protection capability

Table 1. Information for each protective circuit status

\begin{tabular}{cl}
\hline \multicolumn{1}{c}{ Classification } & \multicolumn{1}{c}{ Information } \\
\hline Power & Check for the permitted voltage of the control power \\
\hline Protective circuit A & If the power is normally permitted, Mode 1 (Line A voltage: $+4.4 \mathrm{~V})$, \\
& If the protective line A is damaged, Mode 2 (Line A voltage: $+0.7 \mathrm{~V})$ \\
& If the protective line B is damaged, Line A voltage: $+4.4 \mathrm{~V}$ \\
\hline Protective circuit B & If the power is normally permitted, stand by (Line B voltage: $+0.7 \mathrm{~V})$, \\
& If the protective line A is damaged, Mode 2 (Line B voltage: $+4.4 \mathrm{~V})$, \\
& If the protective line B is damaged, Mode 3 (Line B voltage: $+0.7 \mathrm{~V})$ \\
\hline Confirmation of capability & $\begin{array}{l}\text { If the power is normal and protective line A or B is normal, pulse of }+5 \mathrm{~V} \text { occurs for every 10 } \\
\text { seconds, and if both protective line A and B are damaged (Mode 3), no pulse is generated. }\end{array}$ \\
\hline
\end{tabular}

\subsection{Operation algorithm}

Two protective circuits with same structure and capability are connected in parallel to propose the system to control the switch depending on the circuit condition while one the protective line A is operated. In the event that the protective line A loses the protective capability or having any malfunction, it is transfer for the protective line $\mathrm{B}$, and if the protective line $\mathrm{B}$ loses protective capability, the situation is available to confirm from outside. The operation algorithm is displayed in the following Fig. 3.

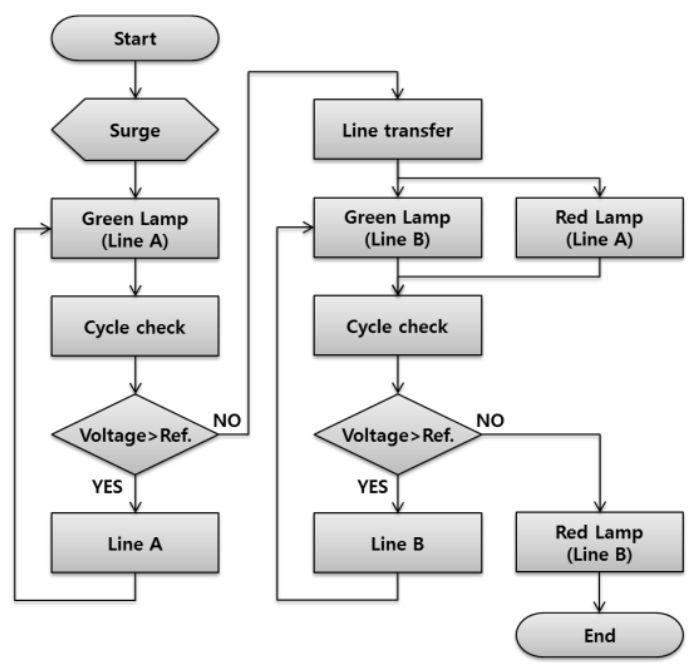

Fig. 3. Operation algorithm.

\section{Experiment and Result}

In order to confirm the capability of the protective system as proposed in this paper, IEC 61643-21 is 
applied to evaluate the characteristics of surge protection, signal transmission and transfer operation [4].

\subsection{Characteristics of surge protection}

The fundamental purpose of the surge protective system, the characteristics of surge protection, is assessed. The experiment circuit is structured as in the following Fig. 4 to carry out the surge current test by the IEC dimension [5], [6].

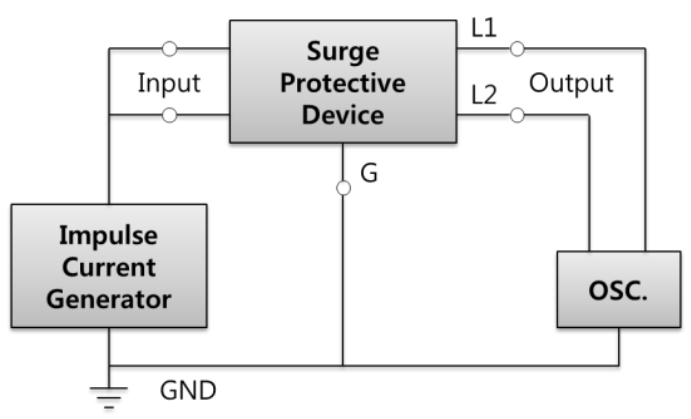

Fig. 4. Experiment circuit of characteristics of surge protection.

The surge device is used to permit the surge current of $8 / 20$ [us], 20[kA] and $10[\mathrm{kA}]$ for straight polarity in between L1-G to check the damages in the protective system as shown in Fig. 5 and there is no erroneous operation or destruction of the protective system.

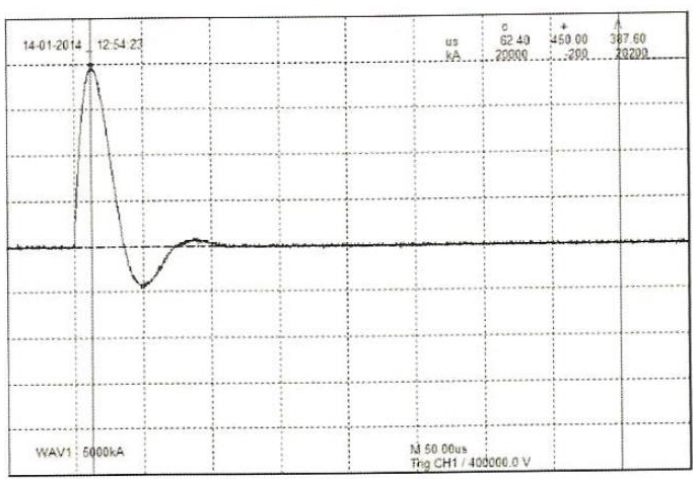

(a)

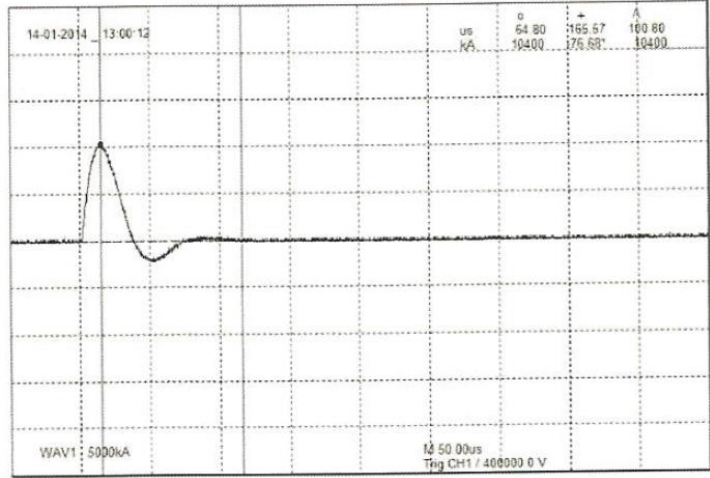

(b)

Fig. 5. Waveform of surge current: (a) 20[kA] and (b) 10[kA].

\subsection{Characteristics of signal transmission}

In order to confirm the characteristics of signal transmission of the protective system, the insertion loss is assessed. As in the following Fig. 6, the experiment circuit is structured to measure the insertion loss [5], [6].

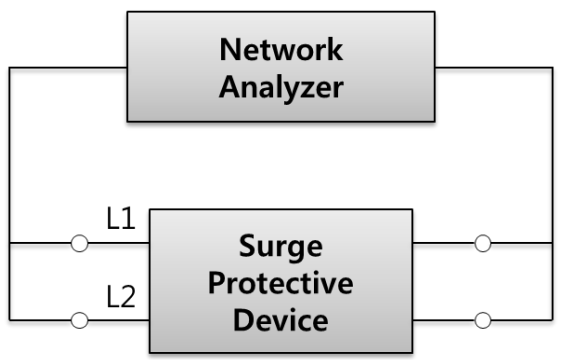

Fig. 6. Experiment circuit of characteristics of signal transmission. 
The network analyzer is used to measure L1-L2 within the frequency range of 5 15[MHz]. As in the following Fig. 7, in the bandwidth of rated frequency of $10[\mathrm{MHz}]$, the insertion loss is measured for $0.293[\mathrm{~dB}]$.

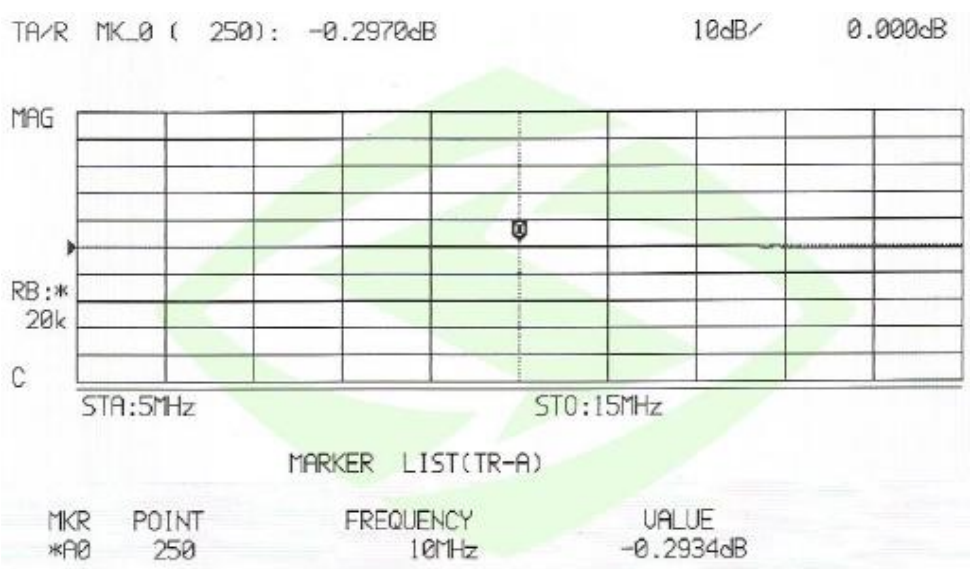

Fig. 7. Experiment circuit of Characteristics of signal transmission.

\subsection{Characteristics of transfer operation}

In order to confirm the transfer characteristics of the protective system, the situation of losing the protective capability is hypothesized. As in the following Fig. 8, in the event that there is any problem in the protective circuit by applying the switch on two protective circuits connected in parallel, the situation for transfer for the circuit by the switch is assessed [7].

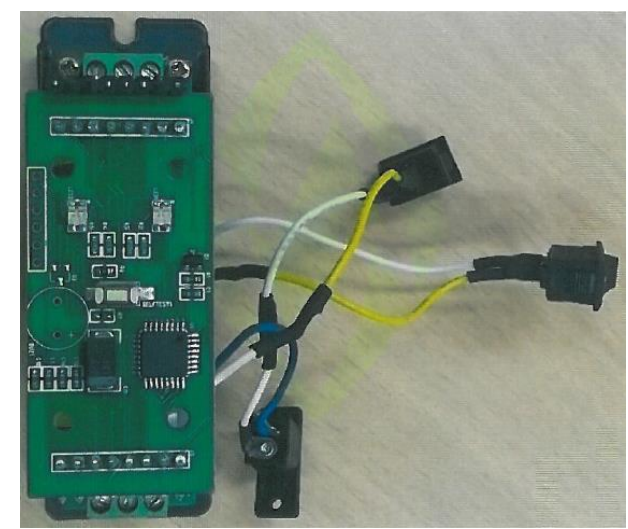

Fig. 8. Photo of experiment sample of characteristics of transfer operation.

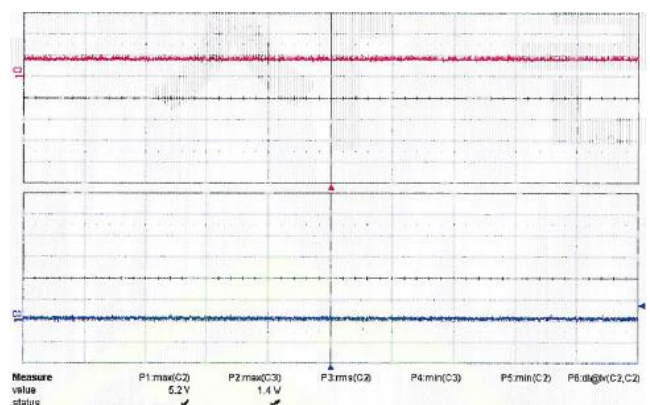

(a)

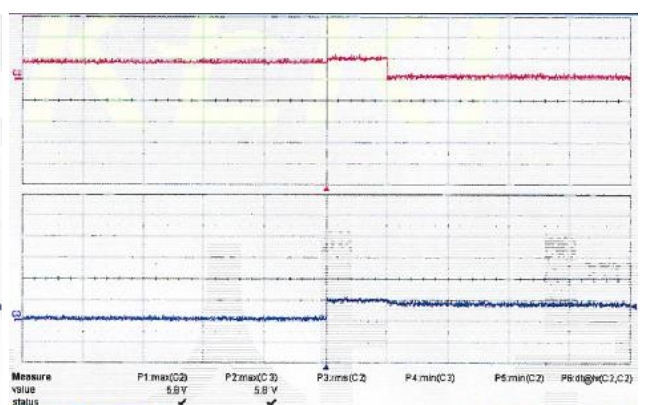

(b)

Fig. 9. Measured waveform of protective circuit transfer time: (a) Basic status and (b) Transfer status. 
When the protective system is normally operated with the protective circuit A, the switch is used to damage the protective circuit A to measure to transfer with protective circuit B within approximately $100[\mathrm{~ms}]$ as shown in Fig. 9.

\section{Conclusion}

In this paper, the parallel structured protective system that is applicable to the DC low voltage with the protective capability on the surge of up to $8 / 20$ [us] and $20[\mathrm{kA}$ ] is proposed, and the experiment evaluation on the characteristics of surge protection, characteristics of signal transmission, and characteristics of transfer operation are carried out.

The proposed protective system is connected in parallel with the same protective circuit for structure and capability and applies the MCU and switch additionally to structure for automatic transfer in accordance with the protective capability of the protective circuit.

When there is any problem to the protective circuit with the voltage of single protective circuit, the transfer is possible to another protective circuit within $100[\mathrm{~ms}]$ as it has the strength to maintain the protective capability while minimizing the loss of the communication track data.

Therefore, as the digital equipment use of inverter, converter, information communication equipment, security system, fire-service preventive facility, solar energy facility and etc. is increased in the future that the utility value would be great as the high-tech surge protective system.

\section{Acknowledgement}

This work was supported by the Technological Innovation R\&D Program (S2270533) funded by the Small and Medium Business Administration(SMBA, Korea).

\section{References}

[1] Smith SB, Standler RB. The effects of surges on electric appliances. IEEE Transactions on Power Delivery, 1992; 7(3):12751282.

[2] Kuwabara N, Koga H, Motomitsu T. A new lightning surge test circuit for telecommunications equipment in Japan. IEEE Transactions on Electromagnetic Compatibility, 1988; 30(3):393-400.

[3] Dong JK, Young SK, Jae JP, Ki SL. Failure rate estimation of MOV for condition monitoring of surge protective devices. The Transactions of the Korean Institute of Electrical Engineers, 2013; 62(9):1302-1307.

[4] Surge Protective Devices Connected to Telecommunications and Signaling networks-Performance Requirements and Testing Methods. IEC 61643-21 (2000).

[5] Protection against Lightning Electromagnetic Impulse, Part1: General Principle. IEC 61312-1 (1993).

[6] Dae-won P, Jae-yong S, Joo-sup H, Gyung-suk K. Development of a surge protective device for computer network to international standards. JKIICE, 2005; 9(6):1253-1259.

[7] Jong YC, Dong JK, Gi SL, Kyung WK. The Development of Parallel Type Surge Protector Device for Communication Line. In: Proc. of Electrical Facilities Fall Conference and General Meeting, KIEE, 2014; 242-243. 